\title{
SCALING PROPERTIES OF EDEN CLUSTERS IN THREE AND FOUR DIMENSIONS
}

\author{
Pierre DEVILLARD* and H. Eugene STANLEY \\ Center for Polymer Studies and Department of Physics, Boston University, Boston, \\ MA 02215, USA
}

\section{Received 7 July 1989}

\begin{abstract}
We study the scaling properties of noise reduced Eden clusters in three and four dimensions for variant $B$ in the strip geometry. We find that the width $W$ for large times behaves as $a(s) g\left(L / s^{d-1}\right)$, where $L$ is the width of the strip, $s$ the noise reduction parameter, $d$ the dimension of space, and $a(s)$ a decreasing function of $s . g$ is a scaling function with the property $g(u) \rightarrow 1 / 2$ as $u \rightarrow 0$ and $g(u) \sim u^{x}$ as $u \rightarrow \infty$, where $\chi$ is the roughness exponent. This scaling result leads to a new way of determining $\chi$. In 3 dimensions, our numerical values for $\chi$ support a recent conjecture by Kim and Kosterlitz: $\chi=2 /(d+2)$, and contradict all the former analytical conjectures. In 4 dimensions, we cannot distinguish between the conjectures of Kim and Kosterlitz and the conjecture of Wolf and Kertész, because large crossovers and finite size effects make the measurement of the exponents difficult.
\end{abstract}

\section{Introduction and motivation}

The surface of the Eden model [1-11] in dimensions greater than 2 has been widely studied and is still a controversial topic. To be specific, consider the strip geometry and start from a hyperplane of width $L$ of seed particles on a hypercubic lattice, with periodic boundary conditions on the sides of the strip. Define the width

$$
w(t, L) \equiv \sqrt{\sum_{i=1}^{N}\left(h_{i}-\bar{h}^{2}\right)},
$$

where $N$ is the number of perimeter sites, $h_{i}$ the height of the $i$ th perimeter site and $\bar{h}$ the mean height,

$$
\bar{h}=\frac{1}{N} \sum_{i=1}^{N} h_{i} \text {. }
$$

Time $t$ is chosen to be the mass of the cluster. Since $\bar{h}=t / L, w$ will be regarded as a function of $\bar{h}$ and $L$ henceforth. The scaling relationship

* This work is based in part on the Ph.D. thesis of Pierre Devillard (Boston University). 


$$
w(\bar{h}, L) j \sim L^{x} f\left(\frac{\bar{h}}{L^{z}}\right)
$$

has been proposed [3], where $\chi$ and $z$ are exponents.

From eq. (3), it follows that there is a scaling region, $L \ll \bar{h} \ll L^{z}$, where

$$
w \sim \bar{h}^{\beta},
$$

with

$$
\beta=\frac{\chi}{z}
$$

For $\bar{h} \gg L^{z}, w(\bar{h}, L)$ saturates to a value proportional to $L^{\chi}$. The exponents $\chi$ and $z$ are known exactly in two dimensions [6],

$$
\chi=\frac{1}{2}
$$

and

$$
z=\frac{3}{2},
$$

so $\beta=1 / 3$.

In higher dimensions, the situation is unclear. Although a scaling relation,

$$
z=2-\chi
$$

has been derived in approximate ways [12-14] and is compatible with previous numerical simulations, there is controversy about the actual value of the exponents $\chi$ and $z$. It has been argued $[6,15,16]$ that, for sufficiently large disorder, eq. (5) holds for all $d$. However it has been recently conjectured [17], on the basis of numerical results, that $\chi$ and $z$ take the values

$$
\chi=\frac{1}{d}
$$

so from (6)

$$
z=2-\chi=\frac{2 d-1}{d}
$$

The analytical solution by Derrida and Spohn [18] of the directed polymer on a Cayley tree indicates that $\beta$ has to go to zero as $d$ tends to $\infty$. On the basis of a functional space renormalization [19], it has been claimed that in 3 dimensions 
$\chi$ takes the value $11 / 30$. Simulations of a restricted solid as a solid growth model have led Kim and Kosterlitz [20] to propose another conjecture,

$$
\chi=\frac{2}{d+2},
$$

the scaling relation (6) being satisfied. Clearly there is a need to determine $\chi$ accurately.

In the Eden model on a lattice, there are two contributions to the width of the interface. The first comes from inclusions and overhangs and is thought to be independent of $L$ [21]; this contribution is called the intrinsic width $w_{\mathrm{i}}$. The second contribution comes from the long wavelength fluctuations of the interface and is the subject of our present interest. As noted in ref. [21], the use of a trick called noise reduction [17,21-23] diminishes $w_{\mathrm{i}}$ and greatly reduces corrections to scaling, making the numerical computation of exponents $\chi$ and $\beta$ apparently easier.

In this paper we propose a new way of determining $\chi$. To this end, we apply noise reduction to variant $B$ of the Eden model $[4,24]$. Open bonds are defined as bonds that join a cluster site to a perimeter site; in variant $B$, an open bond is chosen at random and the corresponding perimeter site is occupied. In our version of noise reduced variant $B$, we place counters on the perimeter sites (not on the open bonds). We denote by $s$ the noise reduction parameter, the nu nber of times a given perimeter site must be chosen before it is actually a sowed to become occupied. The width $w$ now becomes a function of $\bar{h}, L$ and $s$.

Only the active zone of the cluster is stored, and bit coding is used to save memory space. All simulations were carried on an îBM 3090 on $d$-dimensional hypercubic lattices with a $(d-1)$-dimensional substrate. $(L \times L$ substrates for $d=3$ and $L \times L \times L$ substrates for $d=4$.)

\section{Results}

\section{A. L dependence}

We find three regimes:

(i) First, when $\vec{h}$ is less than a few times $L$, we do not get scaling; in this region, when the noise reduction parameter is more than 2 , we found oscillations corresponding to the filling of layers.

(ii) For larger $\bar{h}$ there is a scaling region, $L \ll \bar{h} \ll L^{z}$, where $w \sim \bar{h}^{\beta}$ [see eqs. (4a) and (4b)]. 

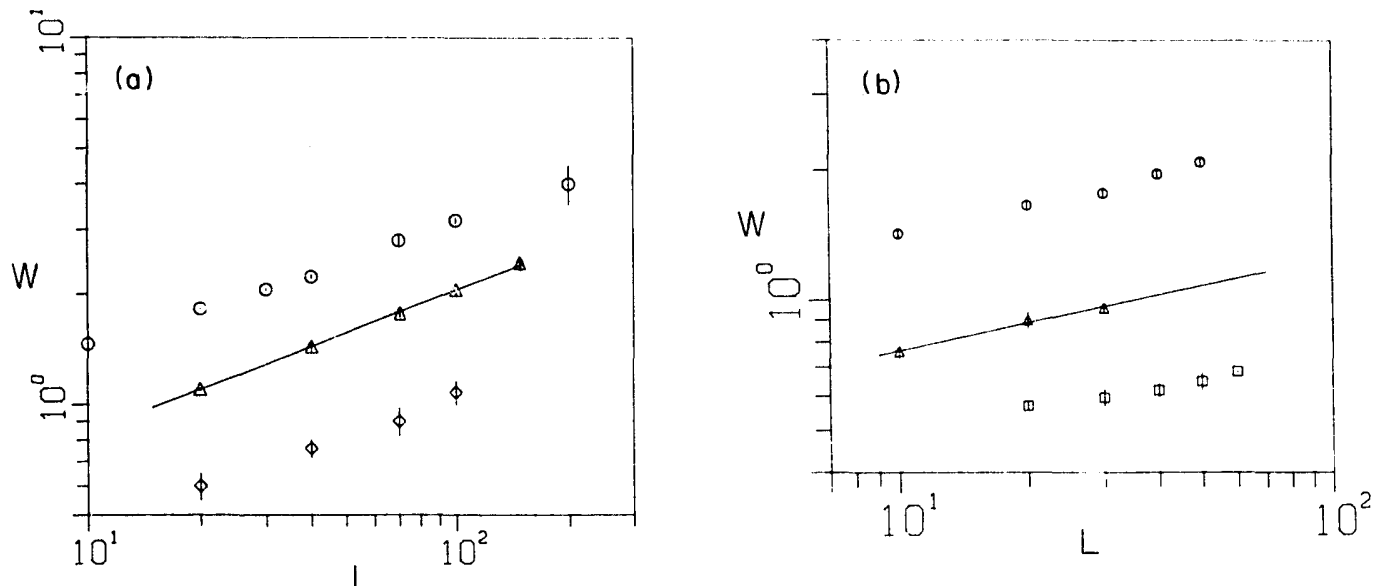

Fig. 1. (a) $\log -\log$ plot of $W$ as a function of $L$ for model B and $d=3$ for various values of the noise reduction parameters: $s=1(O), s=2(\Delta), s=6(\diamond)$. The straight line is a ieast square fit for $s=2$ and $L \geqslant 30$. (b) $\log -\log$ plot of $W$ as a function of $L$ for model B and $d=4$ for various values of $s: s=1(O), s=2(\triangle), s=4(\square)$. The straight line is a least square fit for $s=2$ and $L \geqslant 10$.

(iii) For $\bar{h} \gg L^{z}, w$ saturates and we denote by $W$ the limit of the width as $t \rightarrow \infty$ :

$$
W(L, s) \equiv \lim _{t \rightarrow \infty} w(\bar{h}, L, s) .
$$

Figs. 1a and b show $W$ as a function of $L$ for different values of $s$ for $d=3$ and $d=4$, respectively. For $s=2$, if one tries to fit the data to a form

$$
W(L, s=2) \sim L^{x},
$$

one finds $\chi=0.39 \pm 0.03$ for $d=3$ and $\chi=0.22 \pm 0.03$ for $d=4$. However, we shall show that these results are probably not the actual values of the exponent $\chi$, because of crossover effects. In 3 dimensions, the results were averaged over 4 to 100 samples and in 4 dimensions from 4 to 10 samples.

\section{B. Scaling}

To proceed further, we have to measure the intrinsic width. Following Kertész and Wolf [21] we make the Ansatz

$$
W^{2}=A(s) L^{2 x}+w_{\mathrm{i}}^{2}+\cdots
$$

For $s=1$, we have now three parameters $w_{\mathrm{i}}, A(s=1)$ and $\chi$. We determine these three parameters by minimizing $\left[\log \left(W^{2}\right)-\log \left(A(s=1) L^{2 x}+w_{\mathrm{i}}^{2}\right)\right]^{2}$. We obtained in 3 dimensions $\chi=0.4, A(s=1)=0.26$ and $w_{\mathrm{i}}=0.76$, and in 4 
dimensions, $\chi=0.33, A(s=1)=0.26$ and $w_{\mathrm{i}}=0.92$. We note that the values of $\chi$ obtained here are significantly higher than the values suggested by the raw data. In order to obtain an estimate of the error in $\chi$, we also adopted the following procedure. We fix a value of $\chi$ between 0.1 and 0.6 . For each value of $\chi$, we determine $A(s=1)$ and $w_{\mathrm{i}}^{2}(s=1)$ by minimizing the quantity

$$
C=\sum_{\text {all points }(L, W)}\left[\log W^{2}-\log \left(A(s=1) L^{2 x}+w_{\mathrm{i}}^{2}\right)\right]^{2} .
$$

With the parameters $A(s=1)$ and $w_{\mathrm{i}}^{2}$ obtained, we calculate $C$, which is a measure of how well the points $(L, W)$ are approximated by eq. (10a).

We first take all the experimental points $(L, W)$ at the center of our error bars. $C$ becomes a function of $\chi$, called $C(\chi)$. Then we vary $\chi$ and plot $C(\chi)$ versus $\chi$. We would like to evaluate the influence of the uncertainty in our experimental points on the function $C(\chi)$. We do this by repeating the same procedure as above but, instead of using the center of our error bars, we use the lower bounds of our values of $W$. We obtain a function $C_{\text {low }}(\chi)$. A function $C_{\text {up }}(\chi)$ can be defined in an analogous way, using our upper bounds on our data points.

Fig. 2a shows $C(\chi), C_{\text {low }}(\chi)$ and $C_{\text {up }}(\chi)$ for 3 dimensions. We see that the curves have a minimum in the interval $[0.35,0.46]$. It is tempting to conclude that this leads to a value of $\chi$ in 3 dimensions: $\chi=0.4 \pm 0.06$. The uncertainty, using the above procedure, is large because there is some uncertainty on $w_{\mathrm{i}}^{2}$.

In 4 dimensions, our error bars are much larger than in 3 dimensions. Fig. $2 b$ shows $C(\chi), C_{\text {low }}(\chi)$ and $C_{\text {up }}(\chi)$ for $d=4$. The only thing that can be said, using this procedure, is $0.19<\chi<0.5$.

Fig. 2c shows $W^{2} L^{-2 x}$ as a function of $L^{-2 x}$ in 3 dimensions with the value of $\chi$ obtained previously by the nonlinear least square fit. Fig. $2 d$ shows an analogous plot for $d=4$. Within the error bars, the point fall approximately on a straight line whose slope is $w_{\mathrm{i}}^{2}$ and whose ordinate at the origin is $A(s=1)$.

We define the corrected width as

$$
\bar{W}(L, s)=\left[W^{2}(L, s)-w_{\mathrm{i}}^{2}(s)\right]^{1 / 2} .
$$

We propose the scaling assumption

$$
\bar{W}(L, s)=a(s) g\left(\frac{L}{s^{d-1}}\right),
$$

where $a(s)$ is a function of $s$ and $g$ is a scaling function. In order to test (11), we plot $\bar{W}$ as a function of the scaled variable $L / s^{d-1}$ for values of $L$ up to $L=200$ for $d=3$ (fig. 3a) and $L=80$ for $d=4$ (fig. 3b). We find that within the error 

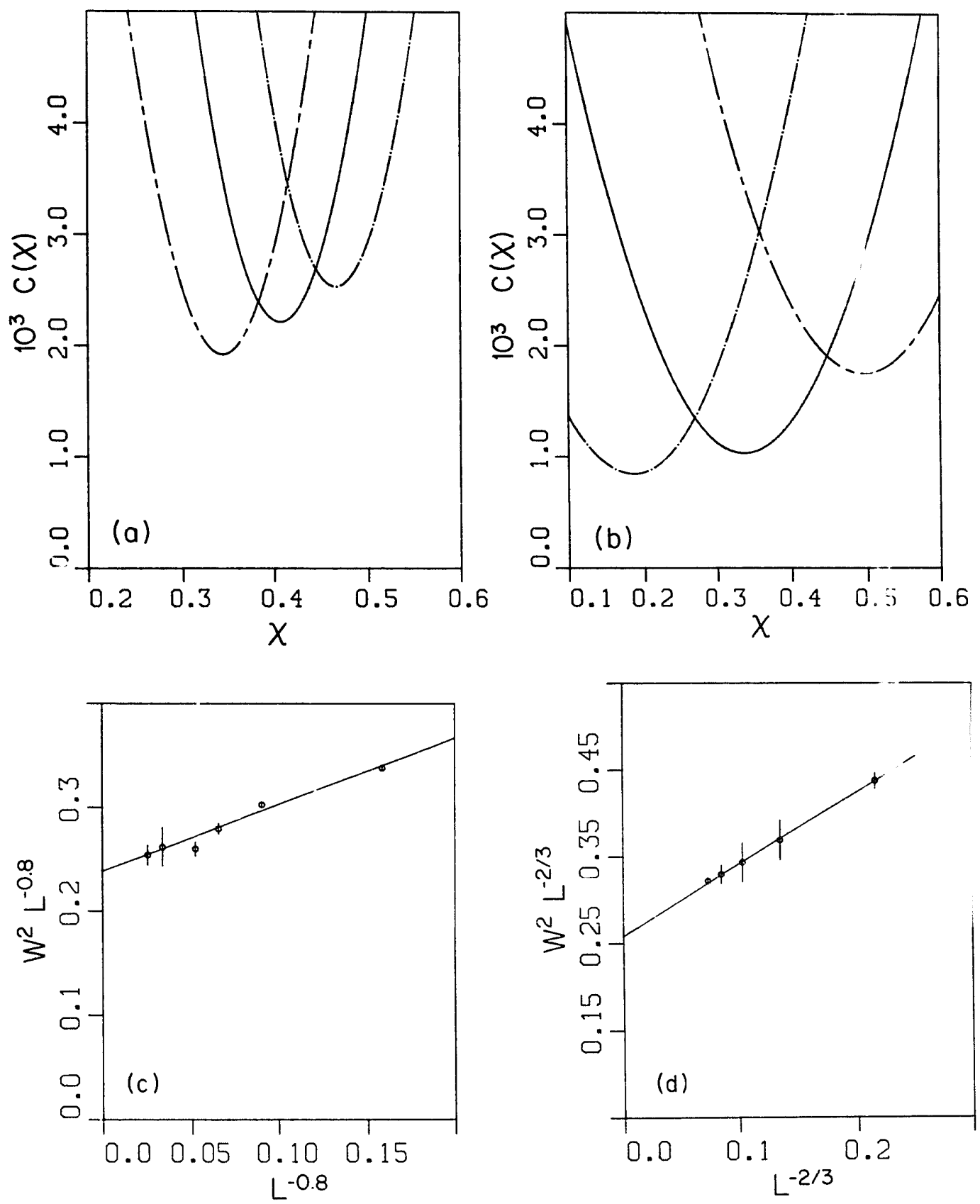

Fig. 2. Plot of $C$ (solid line), $C_{\text {low }}(---)$ and $C_{\text {up }}(--)$ as a function of $\chi$ (a) for $d=3$ and (b for $d=4$. (c) $\log -\log$ plot of $W^{2} / L^{0.8}$ as a function of $1 / L^{0.8}$ for model B and $d=3$ for $s=1$. The straight line is a fit to the data. It gives $w_{\mathrm{i}}=0.8$. (d) $\log -\log$ plot of $W^{2} / L^{2 / 3}$ as a function of $1 / L^{2}$ for model B and $d=4$ for $s=1$. The straight line is a least square fit to the data which gives $w_{\mathrm{i}}=0.9$.

bars, the data fall on the same curve except maybe the data for $s=1$. This means that $a(s)$ is approximately constant for $s \neq 1$. For small values of the argument $u$, our results indicate that the scaling function tends to $1 / 2$ as $g(u)$ tends to zero. This is because for small value $s$ the growth proceeds layer by 

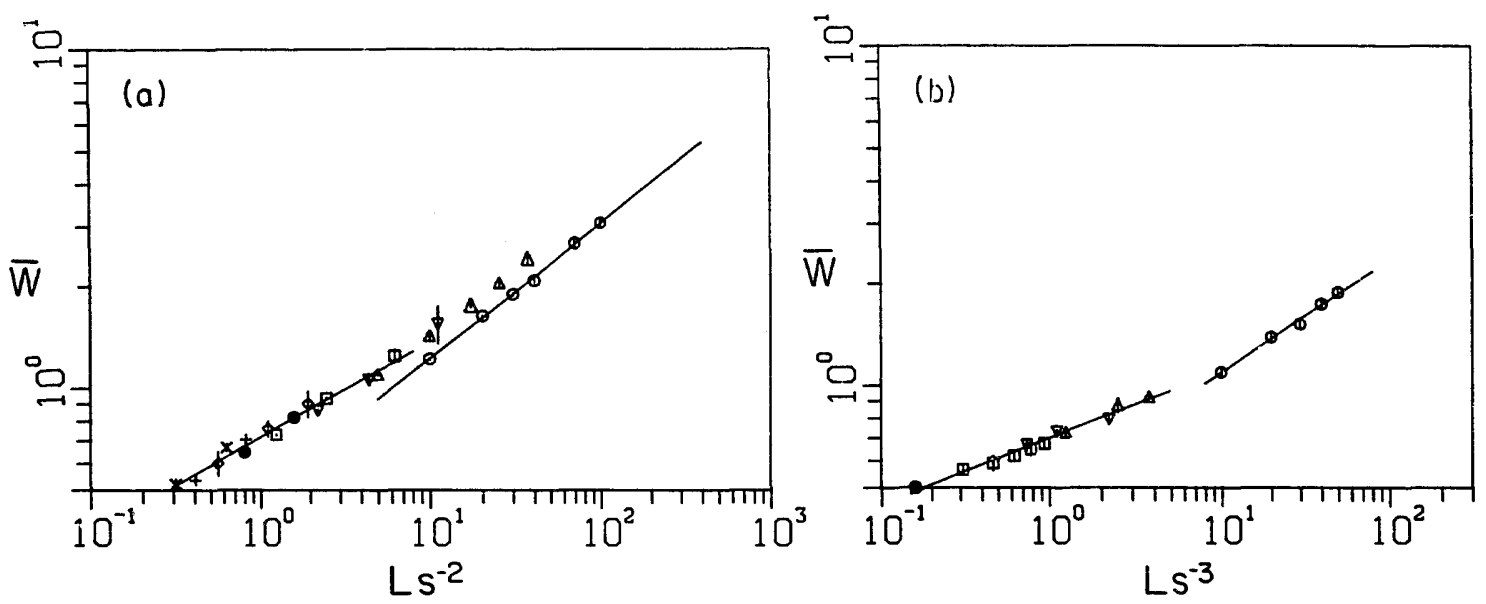

Fig. 3. $\log -\log$ plot of $\bar{W}$ (a) as a function of the scaled variable $L / s^{2}$ for model B for $d=3$ and (b) as a function of $L / s^{3}$ for $d=4$. Here $s=1(\bigcirc), s=2(\triangle), s=3(\nabla), s=4(\square), s=5(\Theta), s=6$ $(\diamond), s=7(+), s=8(\times)$. The upper straight lines are least square fits to the data for which $s=1$ for $d=3$ (a) and $d=4$ (b). The lower straight lines are least square fits to all the data for which $s \neq 1$, (a) for $L / s^{2}<8(d=3)$ and (b) for $L / s^{3}<7(d=4)$.

layer. In the extreme case $s \rightarrow \infty, w(\bar{h}, L, s)$ oscillates between 0 (when $\bar{h}$ is $\left.[n] \times L^{d-1}\right)$ and $1 / 2$ (when $\bar{h}$ is $\left.[n+1 / 2] \times L^{d-1}\right)$. By convention, for $s$ large we take

$$
\bar{W}(L, s)=\lim _{n \rightarrow x} S_{n},
$$

where $S_{n}$ is the maximum value of $w(\bar{h}, L, s)$ for $n \leqslant \bar{h}<n+1$. We found that for fixed $L$

$$
\lim _{s \rightarrow \infty} \bar{W}(L, s)=\frac{1}{2}
$$

The scaling assumption (11) has to break down for very small values of $L$ since $\bar{W}$ is zero for $L=1$. For large values of the argument $u$, we must have

$$
g(u) \sim u^{x},
$$

because of eqs. (3) and (9). We see that the curves $\log \bar{W}$ versus $L / s^{2}$ for $d=3$ have a clear upward curvature (see fig. 3a) for $L / s^{2}<10$. For $L / s^{2}>10$, the curve seems to be straight. A least square fit in the region $s=1,10 \leqslant L / s \leqslant 100$ gives $\chi=0.4 \pm 0.02$. The actual error bars are in fact much larger, because of the uncertainty of the intrinsic width which has been withdrawn. Analogous considerations hold in 4 dimensions. A least square fit to $g(u)$ in the region $L / s^{3}>10$ gives $\chi=0.33 \pm 0.03$. Again, the uncertainty about the intrinsic width at $s=1$ makes the actual uncertainty on $\chi$ much higher than 0.03 . 
Let us suppose that (11) is true and $a(s)$ is approximately constant. If $L$ is fixed and $L / s^{d-1} \gg 1$, then $g\left(L / s^{d-1}\right) \sim\left(L / s^{d-1}\right)^{x}$. Hence

$$
\bar{W}(L, s) \sim s^{-y},
$$

with

$$
y=(d-1) \chi .
$$

Figs. $4 \mathrm{a}$ and $\mathrm{b}$ show $\vec{W}$ as a function of $s$ for different values of $L$ for $d=3$ and $d=4$, respectively. As expected $\bar{W}$ decreases with $s$. We seem to have a region where (14a) is satisfied but in fact our value of the exponent $y$ would give $y=0.65 \pm 0.1$ in 3 dimensions and $y=0.8 \pm 0.1$ in 4 dimensions.

These values do not match (14b) because the largest values of $L / s^{d-1}$ we can achieve with $s \neq 1$ are not large enough for (14a) to hold.

How do these results compare with the scaling form proposed in ref. [22] for variants $\mathrm{A}$ and $\mathrm{C}$ of the Eden model,

$$
w^{2}(\bar{h}, L, s)=\left[B(s) L^{x} F\left(\bar{h} L^{-z}\right) s^{-\xi}\right]^{2}+w_{\mathrm{i}}^{2}(s),
$$

where $F$ is a scaling function, $B$ an $s$-dependent amplitude and $w_{\mathrm{i}}$ an intrinsic width. $\xi$ is an exponent which was found to be approximately 1 for variants $A$ and $C$. Taking the limit $\bar{h} \rightarrow \infty$ in (15a) gives

$$
\frac{\bar{W}^{2}}{L^{2 x}}=B^{2}(s) F_{\varkappa}^{2},
$$
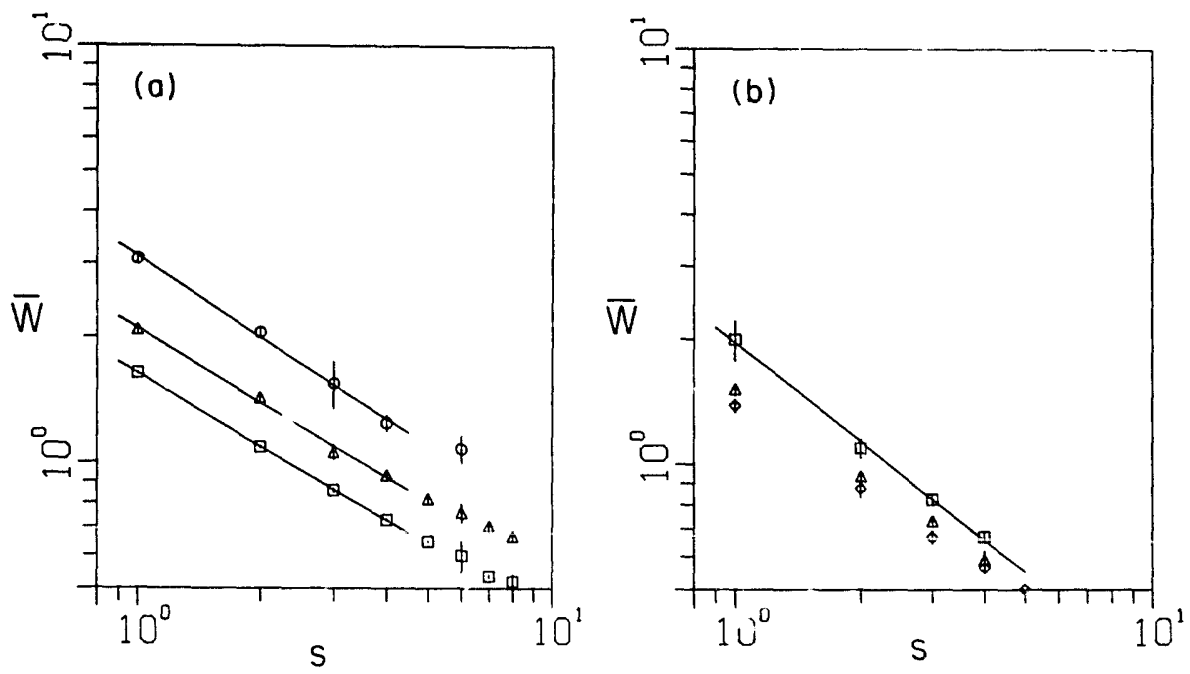

Fig. 4. (a) $\log -\log$ plot of $\bar{W}$ as a function of $s$ for model B and $d=3$ for various values of the width of the strip $L, L=20(\square), L=40(\triangle), L=100(O)$. Straight lines are fits to the data for $L=100, L=40$ and $L=20$, in the interval $1 \leqslant s \leqslant 4$. (b) $\log -\log$ plot of $W$ as a function of $s$ for model B and $d=4$ for various values of $L: L=20(\diamond), L=30(\triangle), L=60(\square)$. The straight line is a it to the data for $L=60$. 


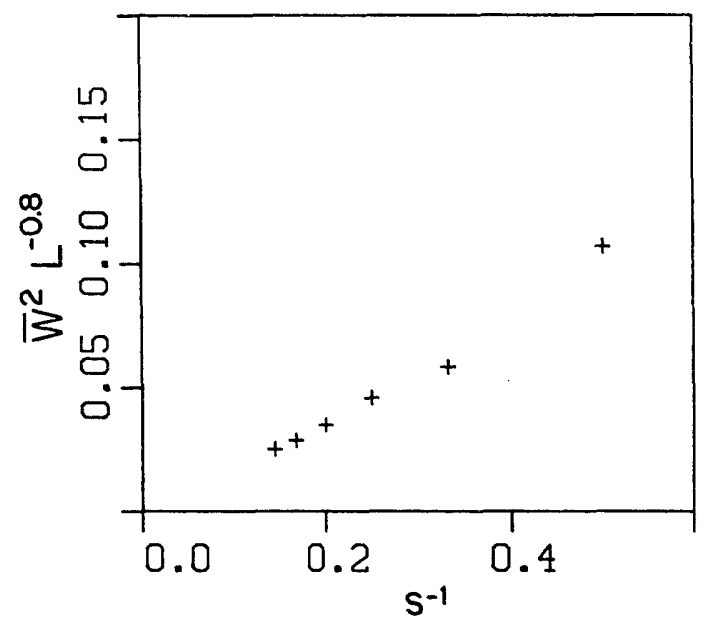

Fig. 5. Plot of $\bar{W}^{2} / L^{0.8}$ as a function of $1 / s$ for model $\mathrm{B}, d=3$ and $L=40$.

with $F_{\infty}=\lim _{x \rightarrow \infty} F(x)$. In two dimensions, for models $A$ and $C, B^{2}$ was found to be of the form $C_{1}+C_{2} / s$. Thus, if (15b) is true,

$$
\frac{\bar{W}^{2}}{L^{2 x}}=\left(C_{1}+\frac{C_{2}}{S}\right) F_{\infty}^{2}
$$

We plotted in fig. $5 \bar{W} / L^{2 x}$ as a function of $1 / s$ for $L=40$ for 3 dimensions with the value $\chi=0.4$ previously obtained. The curve shows a clear upward curvature.

We would like now to see if these data can be better approximated by a form like (11). From (11), we have, for $L \gg s^{d-1}$,

$$
\frac{\bar{W}^{2}}{L^{2 x}} \sim\left(\frac{1}{s}\right)^{2(d-1) x},
$$

and, for $L \ll s^{d-1}$,

$$
\frac{\bar{W}^{2}}{L^{2 x}} \sim \frac{0.25}{L^{2 x}}
$$

If $(11)$ is true, $\bar{W}^{2} / L^{2 x}$ as a function of $(1 / s)^{2(d-1) x}$ should be a straight line for $s \gg s^{*}=L^{1 /(d-1)}$ and go toward a plateau of height $0.25 / L^{2 x}$ for $s \ll s^{*}$. Fig. 6 shows $\bar{W}^{2} / L^{2 x}$ as a function of $(1 / s)^{2(d-1) x}$ for $d=3$ and $L=40$. Although we did not get to large enough $s$ to see the plateau, the portion for $s \leqslant 8$ can reasonably be fitted to a straight line. For $d=4$, our data were not conclusive enough to make the distinction between (15b) and (11). We conclude that, at least in 3 dimensions, for model $B$ with the counters on the perimeter sites, the scaling form (11) seems to be verified rather than eq. (15b). 


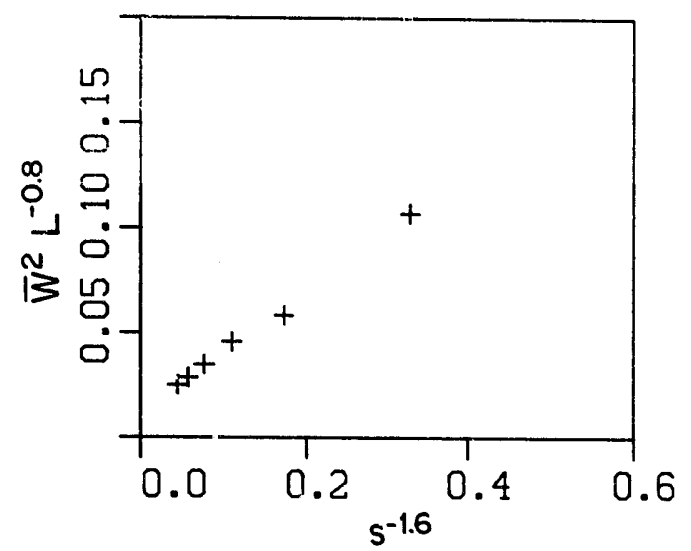

Fig. 6. Plot of $\bar{W}^{2} / L^{0.8}$ as a function of $1 / s^{1.6}$ for model $\mathrm{B}, d=3$ and $L=40$.

\section{Dynamical properties}

(i) $d=3$

We now turn to the evlauation of the dynamical properties. The average height $\bar{h}$ for which the scaling regime begins increases with $s$. This to because noise reduction introduces time rescaling, as found in ref. [22]. Thus, in order to observe the scaling regime we choose $s$ relatively small. Fig. 7a shows $w$ as a function of $\bar{h}$ for $L=400, s=2$ after averaging over 10 samples in 3 dimensions. Since the region where the dynamical scaling relation (4a) is expected is small, we tried to plot the local slope $\beta_{\text {eff }}=\partial \log _{10} w / \partial \log _{10} \bar{h}$ as a function of $\log _{10} \bar{h}[17,22]$. However, this quantity fluctuates a great deal. The interval $-1.5<\log _{10} \bar{h}<2.65$ is then split into boxes $\left[\log _{10} \bar{h}_{n}, \log _{10} \bar{h}_{n+1}\right]$ such that $\bar{h}_{n+1}=4 \bar{h}_{n}$. For the points $\left(\log _{10} \bar{h}, \log _{10} w\right)$ such that $\bar{h}_{n} \leqslant \bar{h}<\bar{h}_{n+1}$, a least square fit to the curve $\log _{10} w$ as a function of $\log _{10} \bar{h}$ gives an average value $\beta_{\mathrm{av}}$ for the interval $\left[\log _{10} \bar{h}_{n}, \log _{10} \bar{h}_{n+1}\right]$. We define $H_{n}$ as $\log _{10} H_{n}=\left(\log _{10} \bar{h}_{n}+\right.$ $\left.\log _{10} \bar{h}_{n+1}\right) / 2$. A plot of $\beta_{\text {av }}$ as a function of $\log _{10} H_{n}$ is shown in fig. 7b. For $H_{n}<0.1, \beta_{\mathrm{av}}$ is roughly $1 / 2$. It decreases, reaches a minimum and then siowly increases. There is a maximum at $H_{n}>30$ and then it decreases again as saturation is approached. The belief that the region $3 \leqslant H_{n} \leqslant 20$ is the scaling region is in our opinion erroneous. The value of $\beta$ should be estimated in the region where $H_{n}$ is larger than 30 , but before it goes down due to saturation effects. For the largest width we could do, we got $\beta=0.22 \pm 0.03$.

(ii) $d=4$

For $d=4$, we averaged over 8 samples with $L=70$ and $s=2$ (see fig. $7 \mathrm{c}$ ). We obtained $\beta=0.116 \pm 0.01$. We also measured $\beta$ for smaller values of $L$ in order to do finite size scaling. A plot of $\beta$ as a function of $1 / L$ is shown in fig. 7d. Finite size effects seem to be particularly important. The $L$ dependence of 

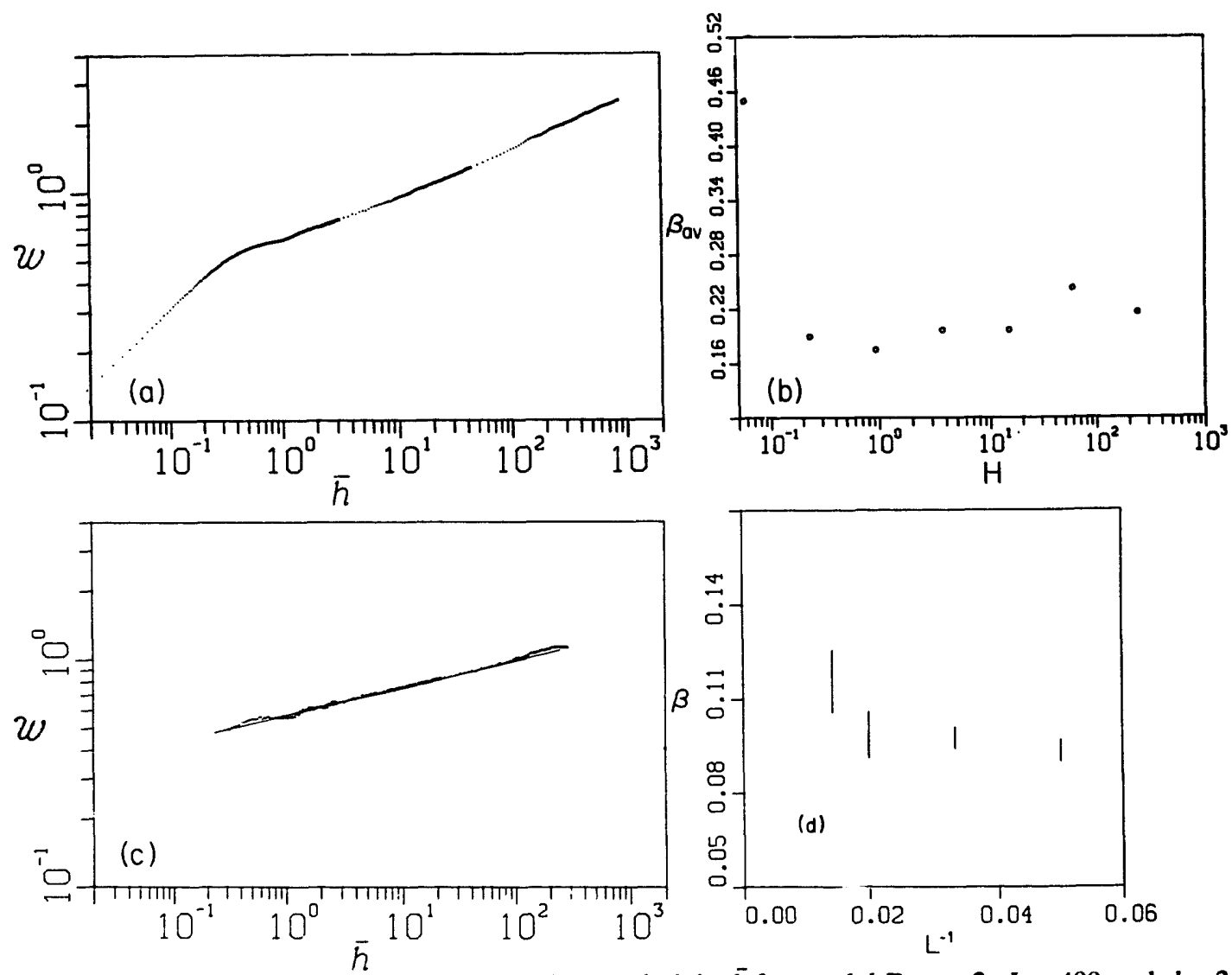

Fig. 7. (a) $\log -\log$ plot of $w$ as a function of mean height $\bar{h}$ for model $\mathrm{B}, s=2, L=400$ and $d=3$, after averaging over 10 samples. (b) Plot of $\beta_{\text {av }}$ as a function of $\log _{10} H$ for the same set of samples. (c) $\log -\log$ plot of $w$ as a function of $\bar{h}$ for model B, $s=2, L=70$ and $d=4$ after averaging over 8 samples. The straight line is a fit in the region $3 \leqslant \bar{h} \leqslant 50$ limited by the two heavy bars. This gives $\beta=0.116 \pm 0.01$. (d) Plot of $\beta$ as a function of $L^{-1}$ for $s=2$ and $d=4$. Extrapolation to infinite $L$ gives $\beta>0.13$.

the effective exponent $\beta$ for finite $L$ is not known. This, together with error bars, renders an extrapolation to $L \rightarrow \infty$ not very reliable.

\section{Discussion and conclusion}

We have studied variant $B$ of the Eden model in three and four dimensions. It responds to noise reduction in a fashion which is different from variants $A$ and $C$ studied in ref. [21]. For variant $B$, with the counters on the perimeter sites, the width for large times obeys the scaling law (10) with

$$
g(u) \sim m^{x}
$$

for large $u$. In 3 dimensions, our numerical resuits for $\chi$ using our values for $s=2$ gives $\chi=0.39 \pm 0.03$. Our values using $s=1$ and subtracting the intrinsic width give $\chi=0.4 \pm 0.06$. These values support a very recent conjecture by 
Kim and Kosterlitz, $\chi=2 /(d+2)$. They seem to contradict the conjectures of refs. $[6,16,17]$. The conjecture of ref. [19] $(\chi=11 / 30)$ is actually near the border of our error bars. In 4 dimensions, finite size effects prevent us from obtaining precise estimates of $\beta$ and $\chi$.

\section{Acknowledgements}

We would like to thank R. Jullien, S. Redner and J. Villain for useful discussions. Very helpful conversations with J. Kertész are gratefully acknowledge. We are also greatly indebted to J.M. Kim and J.M. Kosterlitz for communicating their results to us prior to publication and drawing our attention to the slow approach of the function $g$ to a power law. ine Center for Polymer Studies is supported by grants from the NSF and ONR.

\section{References}

[1] M. Eden, Proc. 4th Berkeley Symp. on Mathematical Statistics and Probability, vol. 4, F. Neyman, ed. (University of California Press, Berkeley, 1961), p. 223.

[2] M. Plischke and Z. Rácz, Phys. Rev. Lett. 53 (1984) 415.

[3] F. Family and T. Vicsek, J. Phys. A 18 (1985) L75.

[4] R. Jullien and R. Botet, J. Phys. A 18 (1985) 2279.

[5] M. Plischke and Z. Rácz, Phys. Rev. A 32 (1985) 3285.

[6] M. Kardar, G. Parisi and Y.C. Zhang, Phys. Rev. Lett. 56 (1986) 889.

[7] M. Plischke, Z. Rácz and D. Liu, Phys. Rev. B 35 (1987) 3485.

[8] D. Dhar, STATPHYS 16, Boston, August 10-16, poster (1986).

[9] J.G. Zabolitzky and D. Stauffer, Phys. Rev. A 34 (1986) 1523.

[10] D. Stauffer, in: Percolation Theory and Ergodic Theory of Infinite Particle Systems, H. Kesten, ed. (Springe ', Heidelberg, 1987).

[11] R. Hirsch and D.E. Wolf, J. Phys. A 19 (1986) L251.

[12] J. Krug, Phys. Rev. A 36 (1987) 5465.

[13] P. Meakin, P. Ramanlal, L.M. Sander and R.C. Ball, Phys. Rev. A 34 (1986) 5091.

[14] E. Medina, T. Hwa, M. Kardar and Y.C. Zhang, Phys. Rev. Lett. 62 (1989) 941.

[15] M. Kardar and Y.C. Zhang, Phys. Rev. Lett. 58 (1987) 2087.

[16] A.J. Mac Kane and M.A. Moore, Phys. Rev. Lett. 60 (1988) 527.

[17] D.E. Wolf and J. Kertész, Europhys. Lett. 4 (1987) 651.

[18] B. Derrida and H. Spohn, J. Stat. Phys. 51 (1988) 817.

[19] T. Halpin-Healy, Phys. Rev. Lett. 62 (1989) 442.

[20] Jin Min Kim and J.M. Kosterlitz, Phys. Rev. Lett. 62 (1989) 2289.

[21] J. Kertész and D.E. Wolf, J. Phys. A 21 (1988) 747.

[22] D.E. Wolf and J. Kertész, J. Phys. A 20 (1987) L257.

[23] C. Tang, Phys. Rev. A 31 (1985) 1977.

J. Nittmann and H.E. Stanley, Nature 321 (1986) 663.

J. Kertész and T. Vicsek, J. Phys. A 19 (1986) L257.

[24] P. Devillard and H.E. Stanley, Phys. Rev. A 38 (1988) 6451.,

[25] R. Jullien and P. Meakin, preprint. 